\title{
Effect of parameter change upon the extra-tropical atmospheric variability
}

\author{
Hanneke E. Levine-Moolenaar • Frank M. Selten • \\ Johan Grasman
}

Received: 19 November 2010/Accepted: 27 September 2011/Published online: 15 October 2011

(C) The Author(s) 2011. This article is published with open access at Springerlink.com

\begin{abstract}
Global climate models contain numerous parameters with uncertain values. In the context of climate simulation and prediction, it is relevant to obtain an estimate of the range of climate outcomes given the parameter uncertainty. Instead of randomly perturbing parameters, we determine parameter perturbations from short-term integrations that potentially have a high impact on the climate of the model. For this purpose we consider a dry, spectral quasi-geostrophic, three-level model on the sphere and its tangent linear and adjoint equations. With an empirical forcing, the model produces a fairly realistic simulation of the extra-tropical winter circulation. We allowed perturbations in a 1,449 dimensional parameter space. As a measure of impact on the climate we compute the change in the probability density function of the dominant patterns of variability. We find that the largest climate response in a set of 1,000 simulations with potentially high impact perturbations is much larger than the largest response in a similar set of simulations with randomly picked perturbations. We conclude that parameter sensitivity calculations based on short term integrations contain valuable information about the sensitivity of the model climate to parameter perturbations. The approach is feasible for stateof-the-art climate models provided that the tangent linear and adjoint equations are implemented.
\end{abstract}

\footnotetext{
H. E. Levine-Moolenaar · F. M. Selten

Royal Netherlands Meteorological Institute, De Bilt,

The Netherlands

H. E. Levine-Moolenaar · J. Grasman ( $₫)$

Biometris, Wageningen University and Research Centre, P.O. Box 100, 6700AC Wageningen, The Netherlands

e-mail: johan.grasman@wur.nl
}

\section{Introduction}

Uncertainty in the outcome of climate models is widely recognized, see Fleming (1993), Allen (2003), Murphy et al. (2004), Stocker (2004), Stainforth et al. (2005) and IPCC (2001). A contributing factor is uncertainty in the model parameters due to parameterization of unresolved physical processes by some approximation. Thus, most of the model parameters are not exactly known so an uncertainty analysis should be part of a study on the long term dynamics of the climate. Of special interest is the range of possible outcomes of the climate model, given the range of parameter uncertainties. A practical method to identify the parameter perturbations that yield the more extreme outcomes is lacking. We refer to these parameter perturbations as high impact parameter perturbations. The aim of this study is to identify those parameter perturbations that have the largest impact on the simulated regional climate given the allowed size of the change in the parameter vector.

Fleming (1993) conducted a preliminary analysis on uncertain model parameters in the context of simple nonlinear dynamical systems. He stated that there is a need to establish a scientific methodology for identifying and quantifying the possible impacts of uncertainty. For complex General Circulation Models (GCMs) he concluded that the Monte Carlo approach is the only practical solution to obtain uncertainty estimates despite the lack of rigor in the determination of the sample size and the enormous computational effort that is required, see also Sambridge and Mosegard (2002). If one is interested in the climate mean response to a small change in forcing, an alternative approach, based on the Fluctuation-Dissipation Theorem (Leith 1975), has been developed, see also Ch. 2 of Majda et al. (2005) for more on this approach. This theorem states that by observing the natural fluctuations of a system with 
certain properties a linear operator can be constructed that gives the response of the system to an external stimulus of sufficiently weak amplitude. The so far most realistic test of the theorem's applicability to the atmosphere is described in Gritsun and Branstator (2007).

The Monte Carlo approach is so costly due to the practical fact that most parameter perturbations have a small effect on the simulated climate and that the impacts of different parameter perturbations do not combine linearly in the regional climate response. Many long term simulations are required to find a good estimate of the range of possible climate outcomes given the parameter uncertainties (Annan and Hargreaves 2007). The Monte Carlo approach can be improved if we could, instead of picking parameter perturbations randomly, choose a priori parameter perturbations that potentially have a high impact on the simulated climate. In this study we calculate from short-term integrations potentially high impact parameter perturbations. These are parameter perturbations that potentially cause a large change in regional climates. Regional climates depend strongly on the frequency of occurrence of preferred atmospheric variability patterns, like for Europe the North Atlantic oscillation (Hurrell 1995; Visbeck et al. 2001) or the European blocking (Carril et al. 2008). As a measure of climate change we therefore evaluate the change in a probability density function (PDF) describing the presence of dominant circulation structures and not just the change in the mean state.

For our investigation we use a dry, spectral quasigeostrophic, three-level model on the sphere (T21QGL3), see Marshall and Molteni (1993). The model is introduced in Sect. 2, along with a description of the measure of climate change that we employ. This measure is based on an evaluation of the probability density function (PDF) of the dominant Empirical Orthogonal Functions (EOFs) of the model. In Sect. 3 the method to calculate potentially high impact parameters from short term integrations is outlined and applied to the T21QGL3 model. The method was developed in Moolenaar and Selten (2004), from now on referred to as MS2004, in the context of the three-component Lorenz model of Rayleigh-Benard convection (Lorenz 1963). The goal of this study is to find out whether this approach also applies to the T21QGL3 model that has much more degrees of freedom $(1,449)$ and produces a fairly realistic simulation of the Northern Hemisphere atmospheric winter circulation (Corti et al. 1997). In Sect. 4 the climate response to potentially high impact parameters is compared to the climate response of randomly picked parameter perturbations and to a particular parameter perturbation that by construction we expect to have large impact on the simulated climate. Section 5 concludes the paper with a discussion of the results.

\section{Description of the model and a measure of climate change}

In the extra-tropics, the atmospheric circulation can be well approximated by the quasi-geostrophic equations. These equations are filtered prognostic equations (gravity waves are absent) and can be written in terms of only one variable, the quasi-geostrophic potential vorticity. Here, the quasi-geostrophic (QG) T21-model is used, which is a spectral, 3-level model, as described by Marshall and Molteni (1993). For the potential vorticity a series expansion in spherical harmonics is made. The time dependent coefficients of this expansion are the state variables of the model. The series of spherical harmonics used in the representation of horizontal fields has a triangular truncation at total wavenumber 21 (T21). The model integrates prognostic equations for the $\mathrm{QG}$ potential vorticity at $200 \mathrm{hPa}$ (level 1), $500 \mathrm{hPa}$ (level 2) and $800 \mathrm{hPa}$ (level 3),

$$
\begin{aligned}
& \frac{\partial q_{k}}{\partial t}=-J\left(\psi_{k}, q_{k}\right)-D_{k}(\psi)+S_{k} \quad \text { with } \\
& J\left(\psi_{k}, q_{k}\right)=\frac{\partial \psi_{k}}{\partial \lambda} \frac{\partial q_{k}}{\partial \mu}-\frac{\partial \psi_{k}}{\partial \mu} \frac{\partial q_{k}}{\partial \lambda}, \quad k=1,2,3,
\end{aligned}
$$

where $q_{k}$ is the potential vorticity (PV) and $\psi_{k}$ the stream function at level $k, J$ the Jacobian in which $\lambda$ represents the longitude and $\mu=\sin (\phi)$ with $\phi$ the latitude. The exact definition of the linear operators $D_{k}$ can be found in Marshall and Molteni (1993). The time-independent but spatially varying potential vorticity source terms $S_{k}$ are empirically determined from observations. They are computed as the average PV tendencies carrying their opposite sign and are obtained by inserting observed stream function fields into a version of (1) in which the PV source terms $S_{k}$ are omitted. This is equivalent to assuming that the sample of observed fields used in such a computation is representative of a statistically stable climatology. Eq. 1 is the vertical discretization of the quasi-geostrophic potential vorticity equation (Holton 1992). In addition to (1) there is also the set of equations that expresses PV in terms of the stream functions:

$$
\begin{aligned}
& q_{1}=\nabla^{2} \psi_{1}-R_{1}^{-2}\left(\psi_{1}-\psi_{2}\right)+f, \\
& q_{2}=\nabla^{2} \psi_{2}+R_{1}^{-2}\left(\psi_{1}-\psi_{2}\right)-R_{2}^{-2}\left(\psi_{2}-\psi_{3}\right)+f, \\
& q_{3}=\nabla^{2} \psi_{3}+R_{2}^{-2}\left(\psi_{2}-\psi_{3}\right)+f\left(1+\frac{h}{H_{0}}\right),
\end{aligned}
$$

where $f=2 \Omega \sin (\phi), R_{1}(=770 \mathrm{~km})$ and $R_{2}(=450 \mathrm{~km})$ are Rossby radii of deformation appropriate to, respectively, the $200-500 \mathrm{hPa}$ layer and the 500-800 hPa layer and $h$ and $H_{0}$ are, respectively, the (real) orographic height and a scale height set to $9 \mathrm{~km}$. At each level the PV has 483 spectral components, so that the model has in total 1,449 degrees of freedom. 
In Corti et al. (1997) it was shown that the T21QGL3 model produces a realistic simulation of the extra-tropical Northern Hemisphere winter circulation including the patterns of low-frequency variability as analysed by an Empirical Orthogonal Function analysis (Preisendorfer 1988). These EOFs form an orthonormal system $\left\{e^{(1)}, e^{(2)}, \ldots\right\}$. The stream function vector $\psi(t)$ has a time mean $\overline{\psi(t)}$. The difference between the two is approximated by the projection upon the first $p$ EOFs:

$\psi(t)-\overline{\psi(t)}=\sum_{i=1}^{p} a_{i}(t) e^{(i)} \quad$ with

$a_{i}(t)=<\psi(t)-\overline{\psi(t)}, e^{(i)}>$,

where $<,>$ denotes the integral over the domain of the Euclidean inner product

$<u, v>=\iint u(\lambda, \phi) \cdot v(\lambda, \phi) \cos (\phi) d \lambda d \phi$.

It defines the norm $|v|=\langle v, v\rangle^{1 / 2}$. In our case $p=483$ when we apply the EOF analysis to the $500 \mathrm{hPa}$ level. The EOFs are the eigen functions of the covariance matrix $(\psi(t)-\overline{\psi(t)})(\psi(t)-\overline{\psi(t)})^{T}$ and the total variance in the stream function equals the sum of the corresponding eigenvalues $\mu_{i}$ as the EOFs are uncorrelated in time

$$
\begin{aligned}
& \iint(\psi(\lambda, \phi, t)-\overline{\psi(\lambda, \phi, t)})^{2} \cos (\phi) d \lambda d \phi \\
& =\sum_{i=1}^{p} \overline{a_{i}^{2}(t)}=\sum_{i=1}^{p} \mu_{i} .
\end{aligned}
$$

The eigenvalues corresponding to the EOFs fall off very quickly. Figure 1 displays the eigenvalues of the first 100 EOFs of the T21QG model calculated from $10^{5}$ daily fields of the $500 \mathrm{hPa}$ stream function over the northern hemisphere. It is observed that most information about the variability is contained in the first leading EOFs. As it is seen in the figure the first 10 EOFs contain $55 \%$ of the information about the variability. By projecting the data along these dominant EOFs and truncating the summation (3) at a certain $n<p$ we can reduce the dimension of the full state space considerably and still retain a good global view of the dynamical range of the system.

The leading EOFs span the space of preferred flow patterns. Figure 2 displays EOF1-EOF4 at the $500 \mathrm{hPa}$ level of the T21QG model with standard parameters as calculated from a sample of $10^{5}$ days. In the T21QG model EOF1 is strongly related to the Atlantic Oscillation (AO) and EOF2 may represent the Pacific North American pattern (PNA). Both patterns are known to have a large influence on regional climates around the Northern Hemisphere (Kerr 2004; Hurrell 1995). We will examine the effect of parameter perturbations on the probability of

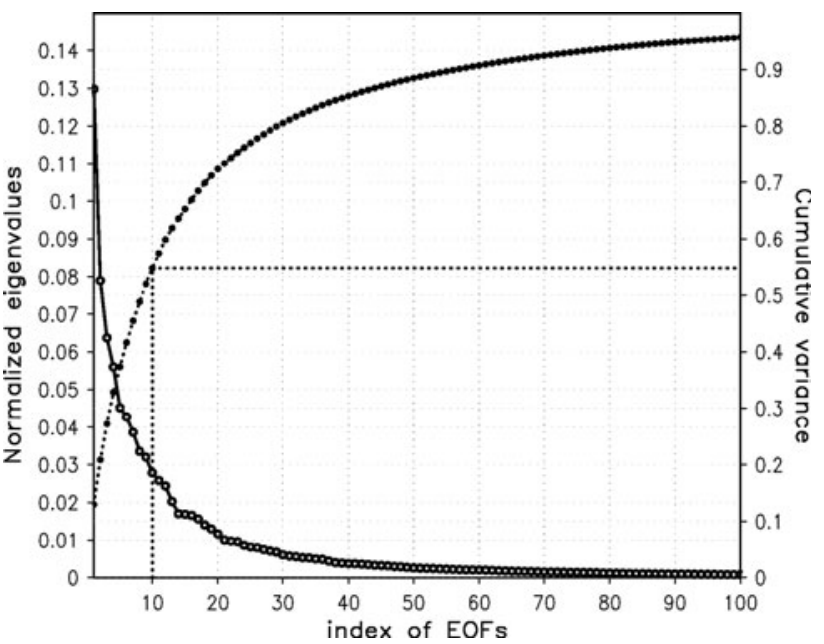

Fig. 1 Eigenvalues $\mu_{i}$ of the first 100 Empirical Orthogonal Functions of the stream function at $500 \mathrm{hPa}$ from a $10^{5}$ day long simulation of the T21QGL3 model. In addition the cumulative variance is given

occurrence of these preferred flow regimes. An important question is formulated as follows: do parameter changes have a notable influence on the probability of occurrence of the preferred flow patterns and therefore on regional climates? Analysis of the changes in the occurrences of the EOFs can give us information about the effect upon regional climates. A climate is characterized by the statistical properties of the circulation over an extended time interval $\left(\sim 10^{5}\right.$ days). The PDFs of the corresponding amplitude time series $a_{i}$ 's of the EOFs, sampled over the $10^{5}$ days long time interval, measure their probability of occurrence. The PDFs are estimated from binned histograms and are shown in Fig. 3 for the first 6 EOFs. The means of these PDFs are zero by construction and the widths of the distributions decrease with the EOF index. The PDF of EOF1 is positively skewed, the others are near to normal. To quantify changes in these PDFs we have to choose a distance measure for two PDFs. The KullbackLeibler distance (Kullback and Leibler 1951) is one of the candidates; see Kleeman (2002) for an application with distributions of the stationary and transient states of the Lorenz equation. In Cha and Srihari (2002) a large number of alternatives can be found. The choice of such a metric may in general affect the outcome when trying to order distributions with respect to their distances. However, in the present case we are dealing with close to normal distributions all having a variance of the same order of magnitude and with a mean and skewness that are highly correlated. It makes that the distributions can very well be approximated by a 1-parameter class of distributions. Consequently, any metric, being a functional that maps the $\mathrm{PDF}$ to a point in $\mathrm{R}^{+}$, will behave orderly. By considering two different metrics, applied to the PDFs as well as to 
their corresponding CDFs, we test the above supposition. First we introduce the parameters $\beta_{i}$ and $\beta_{i}^{c}$ :

$\beta_{i}=\sum_{j=1}^{\text {nbin }}\left\{\operatorname{PDF}_{i}(j)-\operatorname{PDF}_{i}^{(0)}(j)\right\}^{2}$,
$\beta_{i}^{c}=\sum_{j=1}^{\text {nbin }}\left\{\operatorname{CDF}_{i}(j)-C D F_{i}^{(0)}(j)\right\}^{2}$

where $\operatorname{PDF}_{i}(j)$ is the probability of finding $a_{i}$ in bin $j$ for the system with perturbed parameters; $\operatorname{PDF}_{i}^{(0)}(j)$ is the one for the standard parameter values. The goal is to find the largest $\beta_{i}$ within the specified parameter uncertainties because a larger $\beta_{i}$ is expected to give a larger regional climate change. These can be seen as $\mathrm{L}_{2}$-norms. Furthermore, we introduce the parameters

$$
\begin{aligned}
& D_{i}=\max \left[\left|\operatorname{PDF}_{i}(j)-\operatorname{PDF}_{i}^{(0)}(j)\right|, \quad j=1, \ldots, \text { nbin }\right], \\
& D_{i}^{c}=\max \left[\left|\operatorname{CDF}_{i}(j)-\mathrm{CDF}_{i}^{(0)}(j)\right|, \quad j=1, \ldots, \text { nbin }\right]
\end{aligned}
$$

The latter is known as the Kolmogorov-Smirnov test.

\section{Potentially high impact parameter perturbations: methodology based on adjoint systems}

Recent publications suggest that most of the present climate model parameter uncertainty is associated with 'fast physics' (Murphy et al. 2004; Rodwell and Palmer 2007). These processes, at a fast time scale, have an impact on the weather as well as on the climate. Therefore it makes sense to calculate parameter perturbations that have a high impact on short term integrations since these could potentially have a strong impact on the climate as well. This approach was pursued in MS2004 in the context of the Lorenz (1963) model. Here we present a short summary of this approach, followed by the implementation in the T21QGL3 model.

In the three-variable Lorenz system, the evolution of the flow can be depicted by a trajectory in the three-dimensional state space. Starting from a random initial condition the trajectory will settle on the attractor after a certain transient time interval. The attractor is loosely defined by the set of states that the trajectory will visit repeatedly in due course of time. The attractor is chaotic if the solution is
Fig. 2 Preferred circulation pattern for $500 \mathrm{hPa}$ stream function as indicated by EOFs for the T21QGL3 model: a EOF1 related to the $\mathrm{AO}$, b EOF2 related to the PNA, c EOF3 and d EOF4 (a)

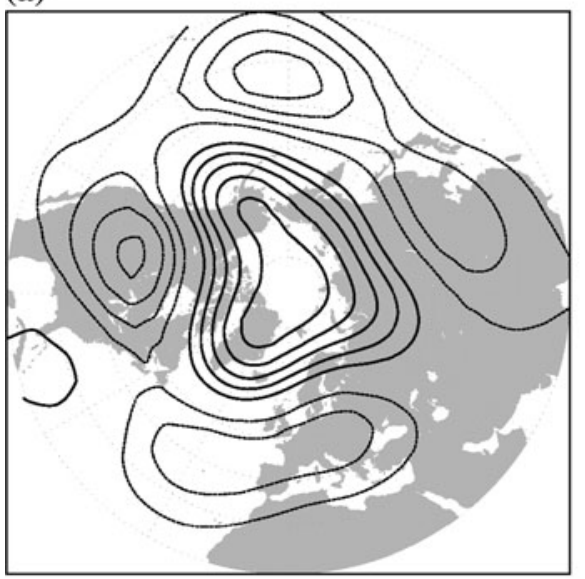

(c)

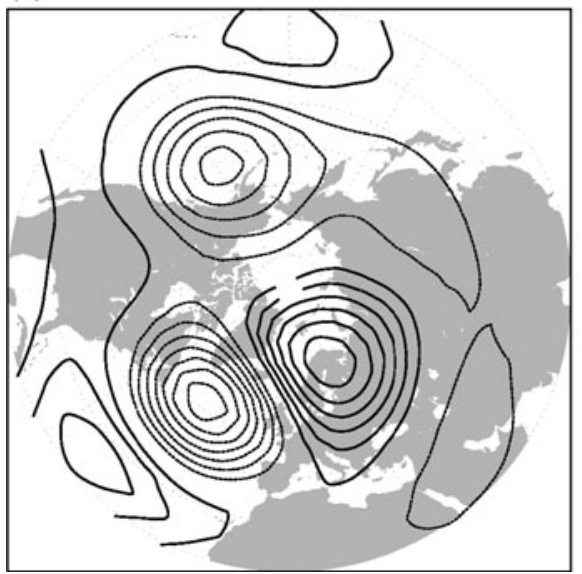

(b)

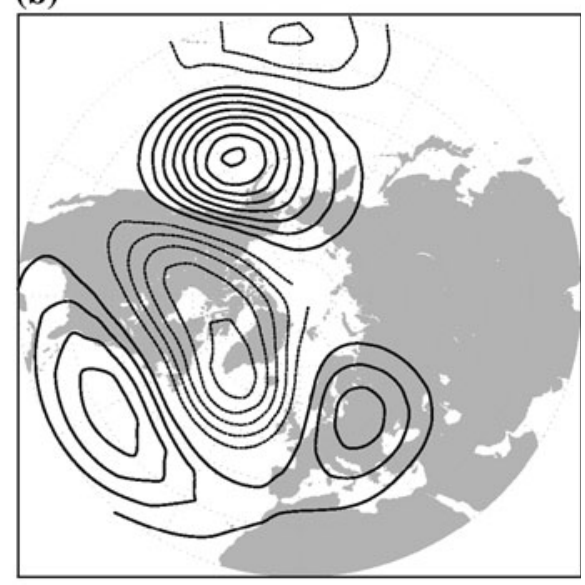

(d)

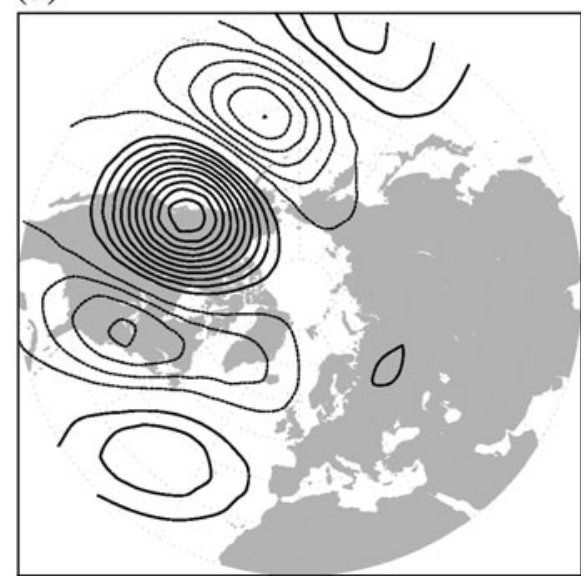




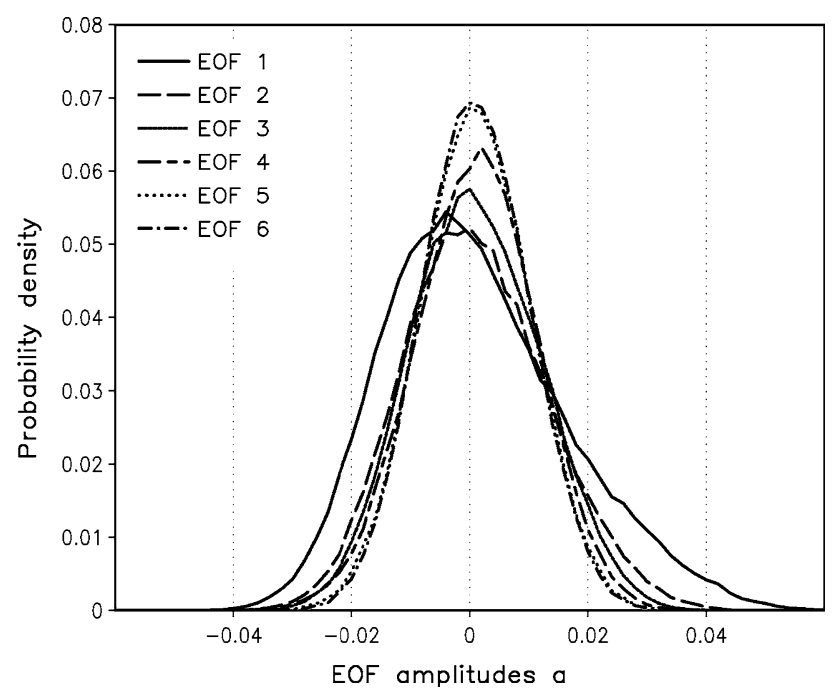

Fig. 3 PDFs of the EOF amplitudes $a_{\mathrm{i}}, i=1, \ldots, 6$. Time means are zero by construction

sensitive to perturbations in the initial condition. This is the case for the standard parameter settings of the Lorenz model. When the parameters are perturbed the attractor changes or in other words, the model climate changes. Starting from an initial condition on the attractor of the unperturbed system the trajectory will settle on the new attractor of the perturbed system after an initial transient period. Given an initial condition we are able to calculate which parameter perturbation within a specified range of uncertainties will have the highest impact on a short-term integration from this initial condition. This parameter perturbation is referred to as the first singular vector. The corresponding singular value is a measure of the magnitude of its impact on the short term evolution. Its structure and its impact depend on the position of the initial state on the attractor, the length of the short term integration and on the particular measure that is chosen to determine the impact on the short term evolution. The study focuses on the dependence of the initial state, while Lea et al. (2000) concentrated on the effect of the length of the integration interval for the Lorenz model. In MS2004 it was found that the singular value varies significantly with initial condition. In other words, not always is the system sensitive to parameter perturbations. It depends on the particular flow configuration of that moment. The main result of the MS2004 study was that parameter perturbations corresponding to singular vectors, calculated from initial conditions just after the trajectory has travelled through a region in state space with high singular values, have potentially a high impact on the model climate. The calculation of the first singular vector can be made in an efficient way, also for high-dimensional systems, when use is made of the so-called tangent linear and adjoint model equations (Barkmeijer et al. 2003). We will take this approach in the context of the T21QGL3 model.

The T21QGL3 model contains numerous uncertain parameters that are given fixed values: the two Rossby radii of deformation, the scale height, the drag coefficient that is a function of location, the temperature relaxation timescale, the diffusion coefficient and the potential vorticity source terms at each level that are also a function of location. For practical reasons we limit the parameter perturbations to the $1,449 \mathrm{PV}$ source terms $S_{k}$. These parameterize the average effect of diabatical processes that at this stage are not explicitly or only crudely modelled like surface heat and momentum fluxes, latent heat release in the atmosphere, convective transports and heating and cooling by radiation. It also includes neglected interactions with unresolved flow structures and topography as well as effects of non-geostrophic components of the flow. As described above, these source terms are empirically determined from observed stream function fields and are inherently uncertain. We computed these terms using daily stream function fields from December, January and February during 1961-1970 and during 1971-1980. We compared both PV source terms by measuring the difference using the Euclidean inner product at each of the three levels and take the sum and normalize this difference by the norm of the PV source terms of the first period. We find that the difference is almost $20 \%$. The uncertainty in the PV source terms is thus considerable. In this study we will consider perturbations to the PV source terms of 5\%, which is probably an underestimate of the real uncertainty, and apply the method of MS2004 to calculate potentially high impact perturbations to the PV source terms. To this end we use an algorithm to calculate so-called 'forcing singular vectors' as devised by Barkmeijer et al. (2003). They compared singular vectors due to a change in initial conditions with singular vectors due to uncertainties in the parametrical vorticity source terms in 2-day forecasts. Then the tangent linear and the corresponding adjoint model need to be extended with equations for the coefficients of the parameter vector $S$. Using Eqs. 1 and 2a we derive the system of differential equations for the stream functions being of the form

$\frac{d \psi}{d t}=F(\psi ; S)$,

where $S$ denotes the PV source terms in the way they turn up in this equation for the stream-function. Next we consider the extended dynamical system

$y=\left(\begin{array}{c}\psi \\ S\end{array}\right)$

Since we assume that the source terms are timeindependent, the tendency equations become: 
$\frac{d y}{d t}=\frac{d}{d t}\left(\begin{array}{c}\psi \\ S\end{array}\right)=\left(\begin{array}{c}F(\psi, S) \\ 0\end{array}\right)=G(y)$,

The tangent linear equations are derived by linearizing Eq. 8 near a non-linear reference orbit $y_{r}$ :

$\frac{d\left(\delta y_{r}\right)}{d t}=J_{G}\left(y_{r}\right) \delta y_{r}$

with Jacobi matrix

$J_{G}\left(y_{r}\right)=\left[\frac{\partial G(y)}{\partial y}\right]_{y_{r}}=\left(\begin{array}{cc}\partial F / \partial r & I \\ O & O\end{array}\right)_{y_{r}}$,

where $O$ is the zero matrix with the appropriate number of rows and columns. The presence of the identity matrix $I$ is understood from the fact that the parameter vector only contains the time-independent PV source terms. The Jacobian matrix $\partial F / \partial r$ is obtained by linearizing Eq. 7 along a reference solution.

The tangent linear equations integrate a small perturbation $\delta y_{r}(0)$ forward in time over a sufficiently short period. This is described by the propagation matrix $R$ :

$\delta y_{r}(T)=R(0, T) \delta y_{r}(0)$.

At initial time the parameter perturbation is set fixed at the unit hypersphere $<\delta S(0), \delta S(0)>=1$ (due to the linearity of the system the size of the perturbation is irrelevant). The initial state $\psi(0)$ is not perturbed, so

$\delta y_{r}(0)=\left(\begin{array}{c}0 \\ \delta S(0)\end{array}\right)=Q \delta S(0)$

with $Q$ an injective mapping ( $Q$ maps $S$ into a larger space). Substitution in (10) yields

$\delta y_{r}(T)=R(0, T) Q \delta S(0)$.

Since we only have to consider the way $\delta \psi$ has evolved at time $T$, we use a surjective mapping $P$ ( $P$ maps vectors back into the smaller, normal state space):

$\delta \psi(T)=P \delta y_{r}(T)=P R(0, T) Q \delta S(0)=M \delta S(0)$.

In summary, integration of Eq. 8 maps vectors $\delta S(0)$ on a unit hypersphere in the 1,449 dimensional parameter space at the initial point $(t=0)$, to a set of vectors $\delta \psi(T)$ given by (13) forming an ellipsoid at the end point $(t=T)$ in the 1,449 dimensional state space of (7).Our aim is to find the parameter perturbation $\delta S$, being constant in time and causing the largest error growth at the end time. This is the vector $\delta S$ that maximizes the ratio

$$
\begin{aligned}
\frac{<\delta \psi(T), N \delta \psi(T)>^{1 / 2}}{<\delta S, N \delta S>^{1 / 2}} & =\frac{<M \delta S, N M \delta S>^{1 / 2}}{<\delta S, N \delta S>^{1 / 2}} \\
& =\frac{<M^{*} N M \delta S, \delta S>^{1 / 2}}{<\delta S, N \delta S>^{1 / 2}}
\end{aligned}
$$

where the matrix $N$ specifies a norm based on the kinetic energy and the matrix $M^{*}$ denotes the adjoint of $M$. Next we consider the following generalized eigenvalue problem

$M^{*} N M \delta S=\lambda N \delta S$

with largest eigenvalue $\lambda$ having an eigenvector called the first singular vector. Using $v=N^{1 / 2} \delta S$ we rewrite (15) as an eigenvalue problem of a symmetric operator:

$N^{-1 / 2} M^{*} N M N^{-1 / 2} v=\lambda v$.

It is solved for the first singular value $\lambda$ and corresponding singular vector $v$ using the Lanczos algorithm (Parlet 1980). The operator $M$ is not explicitly known, it is evaluated by integrating the tangent linear equations. The operator $M^{*}$ follows from a backward integration of the adjoint system (Barkmeijer et al. 2003). We apply this procedure over a short time interval of length $T$ along the reference orbit with a perturbation $\delta S$ to the reference parameter vector $S$ giving the perturbation on the unit hypersphere that causes the largest change in $\delta \psi(T)$. In the next section we describe how this procedure is implemented using a time interval that is large enough to adequately sample the sensitivity of the climate model to parameter perturbations. Moreover, we compare the outcome with that of two alternatives.

\section{Climate response calculations due to differently selected parameter perturbations}

In this section we analyse the response of the T21QGL3 model to various parameter perturbations. First we use the method based on the calculation of singular vectors obtained from the adjoint equations as presented in the previous section in order to produce parameter perturbations that potentially have a high impact. Next we consider randomly chosen parameter perturbations and, finally, we analyse the response of the model to a particular parameter perturbation that we expect to have a large impact by construction.

\subsection{Selecting singular vectors as parameter} perturbations

From a random initial condition we integrated the nonlinear model equations. After a spin up with a length of $10^{3}$ days we consider a time interval of $10^{5}$ days, where the trajectory presumably arrived sufficiently close to the attractor. Next we calculated the first singular value for this reference orbit for the first 5-day interval. We repeated this calculation for time intervals that are 1 day apart. The first singular values of 3505 -day consecutive trajectories are 
plotted in Fig. 4. The first singular value fluctuates considerably as a function of time. Peaks mark the passage of a time interval in which the system is highly sensitive to parameter perturbations. As in the case of the Lorenz model, the sensitivity of the flow to parameter perturbations depends on the exact state of the flow at that moment. In MS2004 it is found that the singular vector calculated from an initial condition of a trajectory that just passed through a sensitive area is likely to be a high impact parameter perturbation. This result is somewhat counterintuitive since one would expect that in time intervals with a large first singular value the system is sensitive to perturbations; particularly in the direction of the corresponding singular vector.

The search for the perturbation vector $\partial S$ that results in the largest climate change is carried out as follows. With the use of the adjoint method parameter perturbations that are likely to have a high impact are selected. The scheme is as follows:

(a) Calculate a short reference orbit over a time interval of 5 days.

(b) Calculate, with the use of the tangent linear and adjoint equations the corresponding first singular vector, along with the first singular value.

(c) Shift the reference orbit 1 day forward and calculate the corresponding first singular vector and the first singular value again.

(d) Look at the evolution of the first singular value and select values that are locally at a minimum after a peak value above a threshold value of 500,000. Then use the corresponding first singular vector as parameter perturbation in a $10^{5}$ day simulation to assess the impact on the climate.

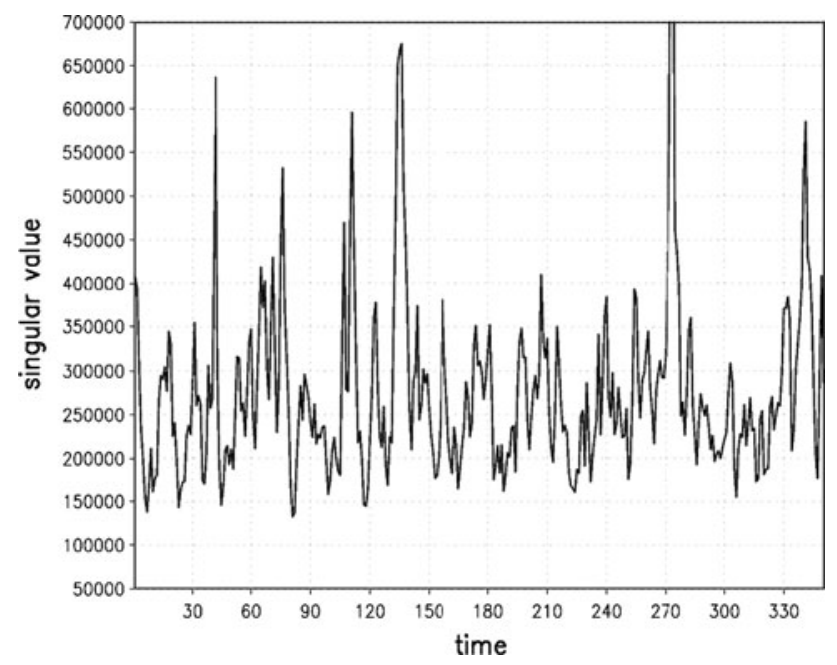

Fig. 4 Evolution of the first singular value of (16) for short integrations of 5 days starting from initial conditions chosen 1 day apart from a 350 day reference trajectory of the T21QGL3 model
One thousand $10^{5}$ day long perturbed integrations of the full nonlinear system (7) are carried out with parameter perturbations according to the singular vectors selected by the above procedure as given by

$\partial S_{k}=0.05 \frac{|S|}{\left|v^{(1)}\right|} v_{k}^{(1)}, \quad k=1,2,3$,

where $v^{(1)}$ denotes such a first singular vector; it is scaled to $5 \%$ of the total PV source term. From each of the in this way perturbed integrations $\mathrm{PDF}_{1}$ and $\mathrm{CDF}_{1}$ of $a_{1}$ are calculated by projecting the stream function upon the first EOF of the unperturbed system. Next the distances to the unperturbed orbit at time $T$ are calculated using the four metrics given by (5) and (6), see Fig. 5 (solid line). In Fig. 5a it is seen that the graph of $\beta_{1}$ for $\mathrm{PDF}_{1}$ falls very rapidly, but there is a long tail consisting of 24 runs with $\beta_{1}>0.02$. The largest value found for $\beta_{1}$ is 0.05 . $\mathrm{PDF}_{1}$ obtained from the singular vector as parameter perturbation direction that gave this largest $\beta_{1}$ is shown in Fig. 7a. The change in the PDF is remarkable: the mean has shifted and the skewness has even changed sign. For the other three parameters we also observe a distribution with a long tail, see Figs. 5b, c, d. Since we are in particular interested in the end part of these tails we give in Fig. 6 from the sample of 1,000 runs the 101 largest changes in the distribution of the amplitude $a_{1}$.

To check whether it is indeed beneficial to select singular vectors as parameter perturbations just after a time interval in which the system is sensitive to parameter perturbation and not at the peak of the sensitivity, we repeated the same procedure but selected the singular vectors at peaks of the singular value above 500,000 as parameter perturbations. We calculated the four distance measures for all 1,000 perturbed $10^{5}$ day simulations and plotted the results in Fig. 5 (dotted line). The largest value found for $\beta_{1}$ is only 0.038 : the probability of values above 0.02 is three times smaller as in the previous case. For the other three parameters a similar behaviour is found. It seems that the results of MS2004 obtained for the Lorenz system carry over to the T21QGL3 model: singular vectors that are selected just after a time interval in which the system is sensitive to parameter perturbations are more likely high impact parameter perturbations than singular vectors selected at peak sensitivity.

\subsection{Random selection of parameter perturbations}

In order to judge the value of our approach of selecting potentially high impact parameter perturbations with the adjoint method, we compare the results with 1,000 randomly chosen parameter perturbations. At each level, we draw randomly a vector from a set of uniformly distributed vectors on the unit hypersphere. Similar to 17 we perturb the standard vorticity source terms $S$ with 5\% in the direction of the randomly chosen vector. Again integrations of $10^{5}$ days are made, 

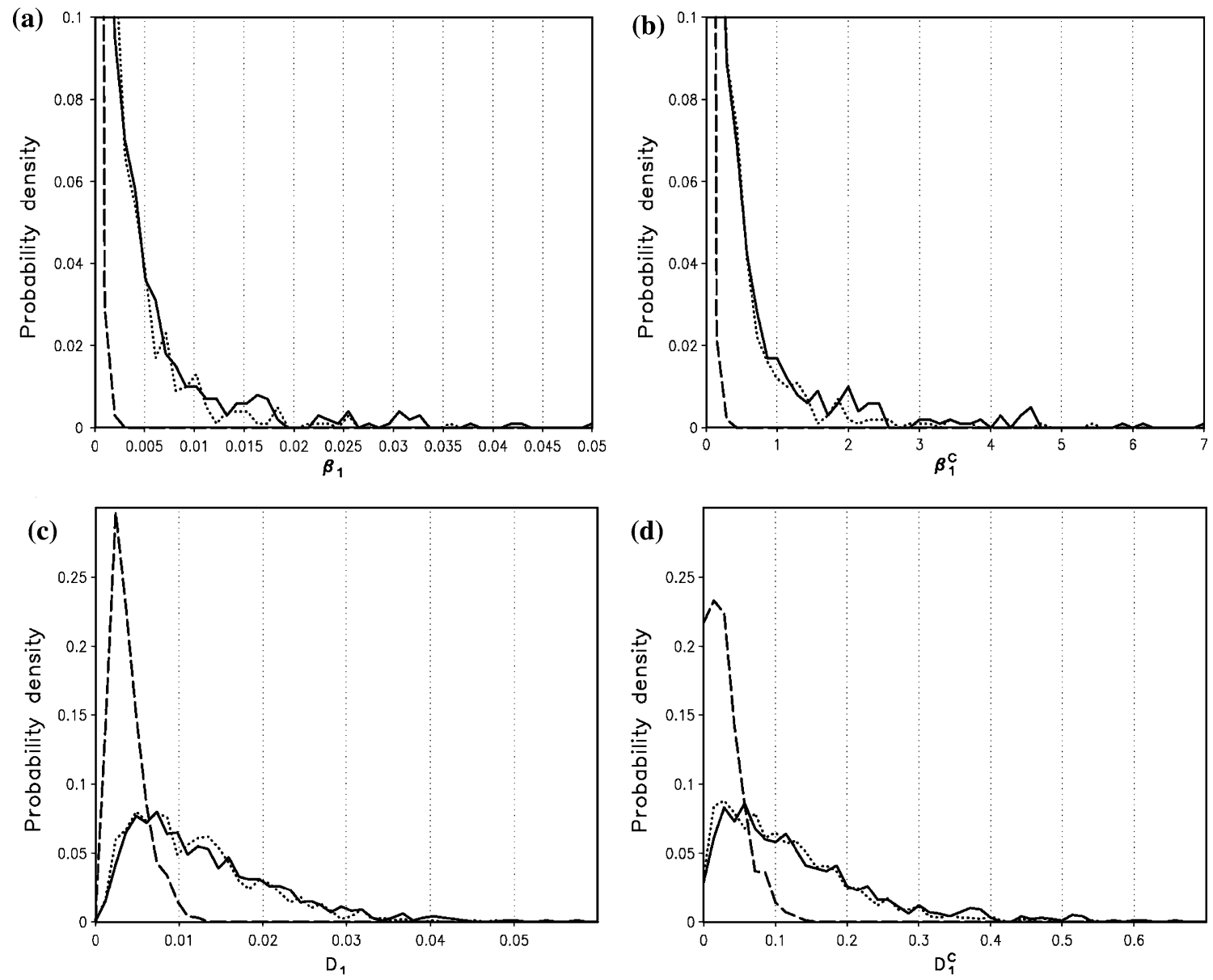

Fig. 5 Distribution of changes in the PDF of the first EOF as defined in $5 \mathrm{ab}$ and $6 \mathrm{ab}$ for a sample of 1,000 perturbed $10^{5}$ day long simulations with perturbed parameters from the adjoint method with direction of perturbation $\partial S$ determined by the singular vector just

after peaks of the first singular value (solid), the singular vector at peaks of the first singular value (dotted) and a random selection of parameter perturbations (dashed) with different distance measures for the PDFs of these amplitude changes: a $\beta_{1}, \mathbf{b} \beta_{1}^{\mathrm{c}}$, c $D_{1}$ and $\mathbf{d} D_{i}^{\mathrm{c}}$

but this time with the randomly chosen parameter perturbations. The PDFs for the four distance measures are shown in Fig. 5 (dashed line). For $\beta_{1}$ the largest value is 0.0025 . This is a factor 20 smaller than the largest $\beta_{1}$ found with the potentially high impact parameter perturbations. In $36 \%$ of the cases, the potentially high impact parameter perturbations yield a larger climate change than this largest climate change found with the selection of random perturbations. $\mathrm{PDF}_{1}$ of the amplitude $a_{1}(t)$ of the perturbed simulation with the random vector corresponding to the largest $\beta_{1}$ is shown in Fig. 7b; the PDF has shifted to positive values and the variance has increased but the changes are modest compared to the most effective parameter perturbation from the adjoint method (Fig. 7a).

\subsection{Parameter perturbation in the direction} of the dominant EOF

The perturbed simulations so far indicate that the response of the PDF of the first EOF dominates the climate response.

In other words, EOF 1 of the T21QGL3 model is most sensitive to perturbations in the PV source terms. Therefore, we expect a large impact if we perturb the PV source terms with a perturbation that has the same geographical pattern as EOF1. A $10^{5}$ day integration was made with perturbed PV source terms at each level with a magnitude of 5\% of the standard PV source terms in the direction of $e^{(1)}$ :

$\partial S_{k}=0.05 \frac{|S|}{\left|e^{(1)}\right|} e_{k}^{(1)}, \quad k=1,2,3$,

so that indeed $|\partial S|=0.05|S|$. It resulted in a PDF1 change of $\beta_{1}=0.016$, a value that is more than 3 times smaller than change due the largest high impact parameter perturbation. To reach the same climate change response of 0.05 as measured by $\beta_{1}$ we need to at least double the strength of the perturbation to $10 \%$. In Fig. 8 we show both PDFs of the perturbed simulations with 5 and $10 \%$ change in the PV source terms in the directions of EOF1. The response turns 

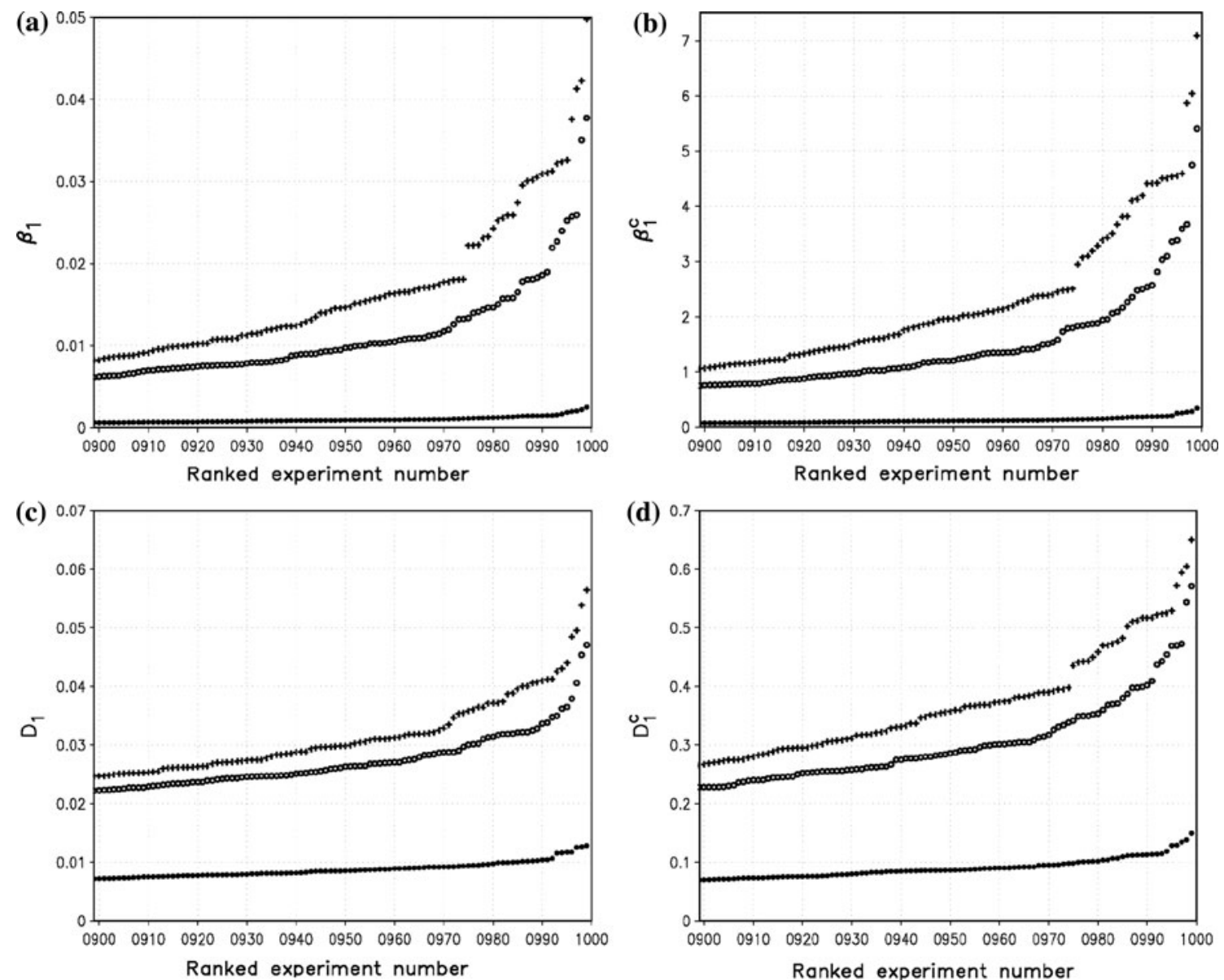

Fig. 6 The largest 101 changes in the amplitude $a_{1}$ from a sample of 1,000 perturbed $10^{5}$ day long simulations with perturbed parameters from the adjoint method with the direction of perturbation $\partial S$ determined by the singular vector just after peaks of the first singular

value (plus), the singular vector at peaks of the first singular value (circle) and a random selection of parameter perturbations (asterisks) for the different norms given by 5-6: a $\beta_{1}$, b $\beta_{1}^{\mathrm{c}}$, c $D_{1}$ and $\mathbf{d} D_{1}^{\mathrm{c}}$
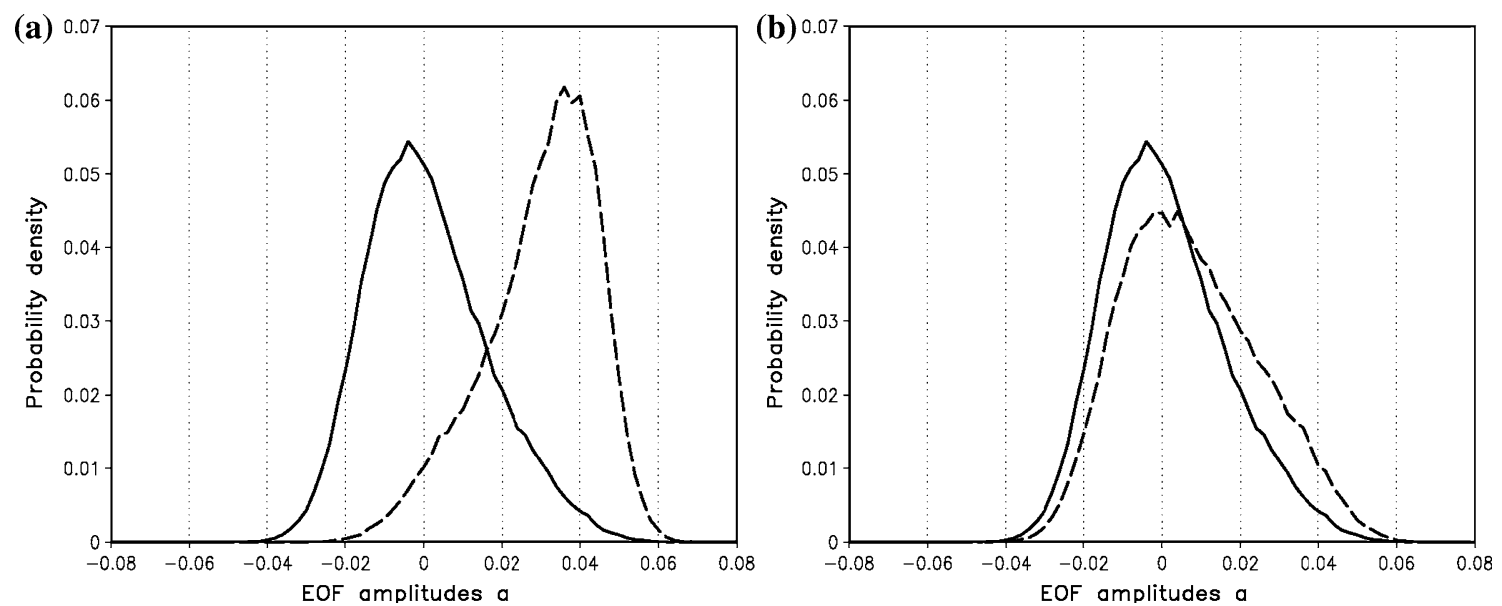

Fig. 7 The solid line represents the PDF of $a_{1}$ for the unperturbed system: a The shifted PDF (dashed) is due to a perturbation in the direction of the singular vector that corresponds to the first singular value just after a peak producing the largest change in the PDF of $a_{1}$

in the norm (5a): $\beta_{1}=0.05$. b The shifted PDF (dashed) is due to the perturbation in the sample of 1,000 random perturbations that gives the largest change in $a_{1}: \beta_{1}=0.0025$ 


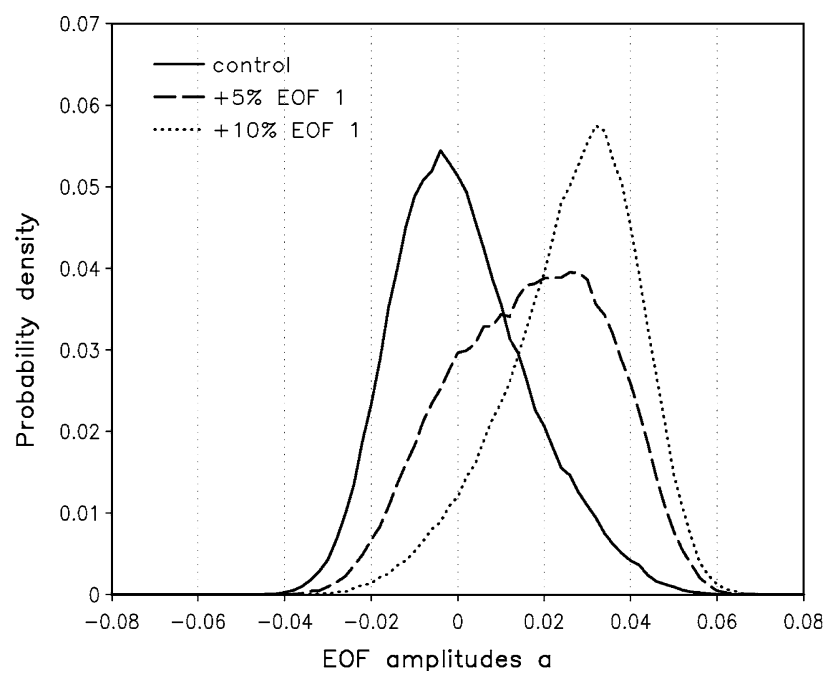

Fig. 8 PDF1 of $a_{1}$ from the unperturbed integration (solid), the perturbed integration with a $5 \%$ perturbation of the PV source term in the direction of EOF1 (dashed) with $\beta_{1}=0.016$ and a $10 \%$ perturbation (dotted)

out to be non-linear: 5\% of the PDF shifts to more positive values, while the variance increases and the skewness decreases. At $10 \%$ the PDF shifts further, the variance decreases again and the skewness increases.

\section{Conclusions}

The subject of this study is the development of a method that can be applied to climate models in order to find, within a specified uncertainty range, perturbations in the model parameters that yield the largest change in the climate statistics. To determine this change, a climate model has to be integrated over a sufficiently large time interval of the order $10^{5}$ days. Since a long computer time is required to evaluate the effect of a new set of parameter values, standard methods based on random selection of parameter perturbations, that need a lot of such evaluations cannot produce within a reasonable time span the maximum climate change possible because of the large dimension of the parameter vector. In a related study Moolenaar and Selten (2004) developed a method to calculate potentially high impact parameter perturbations on the basis of the sensitivity of short integrations to parameter perturbations. The method was developed in the context of the three-variable Lorenz model (Lorenz 1963).

In this study we evaluate this method for a much more realistic model of the extra-tropical atmosphere, the T21QGL3 model of Marshall and Molteni (1993). We have chosen as measure of climate change the response in the PDF of the dominant EOFs because the circulation structures captured by these EOFs have a large impact on regional climates. Using the extended tangent linear and adjoint equations of the model, we have found that a given fraction of the potentially highest impact parameter perturbations lead to a larger climate change than the maximum climate change found from the same fraction based on a purely random selection of parameter perturbations. The result will depend on the chosen metric when estimating the change in the PDF (or CDF) of the EOF amplitude before and after the perturbation. We considered four metrics which lead all the same conclusion. The largest climate change found on the basis of the potentially highest impact parameter perturbations was much larger than the one found on the basis of random parameter perturbations. Table 1 summarizes the maximum climate change found for the different selection methods of parameter perturbations in this study. It is noted that the adjoint method variant that picks the singular vector just after a peak in the largest singular value performs the best. From the tails of the PDFs of $\beta_{1}$ (Fig. 5a) we deduce that this is not just a lucky shot: the probability of a climate change large than a $\beta_{1}$ value of 0.02 is three times larger than for the adjoint method variant with a selection of the singular vector at a peak of the first singular value. When using one of the other three metrics a difference in the same order is found.

The way we analysed large impact changes in the parameter vector makes it possible to identify sensitive combinations of parameters in the process of tuning these parameters. In addition, it may give more insight in the sensitive interaction of different processes in the climate system. As already mentioned the method originates from a systematic analysis on the effect of parameter perturbations in the Lorenz equations (Moolenaar and Selten 2004). In retrospect the clue why the method works lies probably in the higher sensitivity of the system at the moment it is in its

Table 1 Maximum climate change found in sets of 1,000 perturbed simulations for different methods of selecting the parameter perturbation within the specified uncertainty range of $5 \%$ for the four metrics given by $5-6$

\begin{tabular}{lllll}
\hline Perturbation type $(\partial S)$ & $\operatorname{Max}\left(\beta_{1}\right)$ & $\operatorname{Max}\left(\beta_{1}^{\mathrm{c}}\right)$ & $\operatorname{Max}\left(D_{1}\right)$ & $\operatorname{Max}\left(D_{1}^{\mathrm{c}}\right)$ \\
\hline In direction of EOF1 & 0.016 & 2.2 & 0.031 & 0.38 \\
Adjoint method (SV at peak) & 0.038 & 5.4 & 0.047 & 0.57 \\
Adjoint method (SV after peak) & 0.050 & 7.1 & 0.056 & 0.013 \\
Random & 0.0025 & 0.34 & 0.14 \\
\hline
\end{tabular}


most unstable state being a peak in the first singular value. A similar phenomenon is found for the perturbation sensitivity of stable solutions of dynamical systems depending on their parameters: a high sensitivity occurs near a socalled tipping point, see Dakos et al. (2008) and Scheffer et al. (2009). The fact that choosing the moment just after the peak slightly improves the result is secondary; an explanation for this is not yet at hand.

Although we do not exclude the possibility that in the future more effective methods will be developed, we now have brought up a method that may create more openings in the field of uncertainty analysis of highly nonlinear systems. Moolenaar et al. (2007) already carried out a sensitivity analysis for climate driven ecological systems with respect to the ecological parameters using the adjoint method of this study. These parameters are also present in terms that are nonlinear in the state variables being comparable with terms in climate models with products of state variables. This is a situation that cannot be handled with uncertainty analysis methods based on a linearity assumption in case of small system perturbations. Although the adjoint equations become slightly more complex, the approach still continues to hold. The method of this study is applicable to current state-of-the-art global circulation models for studying the dependence on parameters that are more directly linked to specific, parameterized physical processes, like convection, precipitation, clouds and radiation.

Acknowledgements The authors thank J. Barkmeijer (KNMI) for allowing us to use his code to calculate the forcing singular vectors. They also thank J. Greenaway (ECMWF) for helping to run the model at the ECMWF computing facilities.

Open Access This article is distributed under the terms of the Creative Commons Attribution Noncommercial License which permits any noncommercial use, distribution, and reproduction in any medium, provided the original author(s) and source are credited.

\section{References}

Allen MR (2003) Possible or probable? Nature 425:242

Annan JD, Hargreaves JC (2007) Efficient estimation and ensemble generation in climate modeling. Phil Trans R Soc A 365:2077-2088

Barkmeijer J, Iversen T, Palmer TN (2003) Forcing singular vectors and other sensitive model perturbations. Q J Meteor Soc 129:2401-2424

Carril AF, Gualdi S, Cherchi A, Navarra A (2008) Heatwaves in Europe: areas of homogeneous variability and links with the regional to large-scale atmospheric and SSTs anomalies. Clim Dyn 30:77-98

Cha S-H, Srihari SN (2002) On measuring the distance between histograms. Pattern Recogn 35:1355-1370

Corti S, Giannini A, Tibaldi S, Molteni F (1997) Patterns of lowfrequency variability in a three-level quasi-geostrophic model. Clim Dyn 13:883-904
Dakos V, Scheffer M, Van Nes EH, Brovkin V, Petoukhov V, Held H (2008) Slowing down as an early warning signal for abrupt climate change. Proc Natl Acad Sci 105:14308-14312

Fleming RJ (1993) The dynamics of uncertainty: application to parametrization constants in climate models. Clim Dyn $8: 135-150$

Gritsun A, Branstator G (2007) Climate response using a threedimensional operator based on the fluctuation-dissipation theorem. J Atmos Sci 64:2558-2575

Holton JR (1992) An introduction to dynamic meteorology, 3rd edn. Academic Press, London

Hurrell JW (1995) Decadal trends in the North Atlantic Oscillation: regional temperatures and precipitation. Science 269:676-689

IPCC (2001) Climate change: the scientific basis. Contribution of Working Group I, Third Assessment Report. Cambridge University Press, Cambridge

Kerr RA (2004) A few good climate shifters. Science 306:599-601

Kleeman R (2002) Measuring dynamical prediction utility using relative entropy. J Atmos Sci 59:130-141

Kullback S, Leibler RA (1951) On information and sufficiency. Ann Math Statis 22:79-86

Lea DJ, Allen MR, Haine TWN (2000) Sensitivity analysis of the climate chaotic system. Tellus 52A:523-532

Leith CE (1975) Climate response and fluctuation dissipation. J Atmos Sci 32:2022-2026

Lorenz EN (1963) Deterministic nonperiodic flow. J Atmos Sci 20:130-141

Majda A, Abramov MJ, Grote J (2005) Information theory and stochastics for multiscale nonlinear systems. CRM Monograph Series, vol 25

Marshall J, Molteni F (1993) Towards a dynamical understanding of planetary-scale flow regimes. J Atmos Sci 50:1792-1818

Moolenaar HE, Selten FM (2004) Finding the effective parameter perturbations in atmospheric models: the Lorenz63 model as case study. Tellus 56A:47-55

Moolenaar HE, Grasman J, Selten FM, De Gee M (2007) Testing a method for analyzing the effect of parameter change in climate driven ecological systems. Ecol Model 205:289-300

Murphy JM, Sexton DMH, Barnett DN, Jones GS, Webb MJ, Collins M, Stainforth DA (2004) Quantification of modelling uncertainties in a large ensemble of climate change simulations. Nature 430:768-772

Parlet BN (1980) The symmetric eigenvalue problem, series in computational mathematics. Prentice Hall, Englewood Cliffs

Preisendorfer RW (1988) Principal component analysis in meteorology and oceanography. Elsevier Science, Oxford

Rodwell MJ, Palmer TN (2007) Using numerical weather prediction to assess climate models. Q J R Meteor Soc 133:129-146

Sambridge M, Mosegard K (2002) Monte Carlo methods in geophysical inverse problems. Rev Geophys 40. doi:10.1029/ 2000RG000089

Scheffer M, Bascompte J, Brock WA, Brovkin V, Carpenter SR, Dakos V, Held H, Van Nes EH, Rietkerk M, Sugihara G (2009) Early-warning signals for critical transitions. Nature 461:53-59

Stainforth DA, Aina T, Christensen C, Collins M, Faull N, Frame DJ, Kettleborough JA, Knight S, Martin A, Murphy JM, Piani C, Sexton D, Smith LA, Spicer RA, Thorpe AJ, Allen MR (2005) Uncertainty in predictions of the climate response to rising levels of greenhouse gases. Nature 433:403-406

Stocker TF (2004) Models change their tune. Nature 430:737-738

Visbeck M, Hurrel JW, Polvani L, Cullen HM (2001) The North Atlantic oscillation: past, present, and future. Proc Natl Acad Sci 98:12876-12877 